\title{
Head Injuries at the Emergency Department of a University Hospital in Kathmandu
}

\author{
Thérèse Henriksson ${ }^{1}$, Jacob Kjellberg ${ }^{1}$, Yogendra Shakya², Göran Kurlberg ${ }^{1}$ \\ ${ }^{1}$ Department of Surgery, Sahlgrenska Academy, University of Gothenburg, Sweden ${ }^{2}$ General Practice and \\ Emergency Medicine, Maharajgunj Medical Campus, Tribhuvan University Teaching Hospital, Institute of \\ Medicine, Kathmandu, Nepal
}

\section{Corresponding author:}

\section{Thérèse Henriksson, MBBS}

Department of Surgery, Sahlgrenska Academy, University of Gothenburg, Sweden

Email: theresehenriksson@live.se

Submitted : Sep 9, 2020

Accepted : Dec 3, 2020

\begin{abstract}
Introduction

Traumatic head injuries constitute a major cause of morbidity and mortality worldwide. The developing world is particularly affected due to a high prevalence of risk factors and difficulties in enforcing preventive efforts. This study was carried out at the Emergency Department (ED), Tribhuvan University Teaching Hospital (TUTH), Kathmandu, Nepal - where head injuries account for five per cent of all emergency visits. The aim was to describe demographics, cause of trauma, type of injury, and severity according to the Glasgow Coma Scale (GCS), as well as the outcome of emergency attendance in patients seeking medical care for traumatic head injuries. Auxiliary aims were to study correlations between age, cause of trauma and injury severity.
\end{abstract}

\section{Methods}

Data from 577 cases of head injuries from June to October 2019 were collected retrospectively.

\section{Results}

Traumatic head injuries accounted for $5.2 \%$ of all emergency visits. The median age was 23 years. Patients were predominantly male. Superficial injuries and concussions were the most common. Most injuries were mild $(94.4 \%$, GCS $13-15)$ and caused by falls (51.3\%) or road traffic accidents (19.1\%). Fall accidents and mild injuries had the lowest median age. Patients from outside Kathmandu Valley constituted $44.9 \%$ of the cases. Only $12.1 \%$ of the patients were admitted to the hospital.

\section{Conclusion}

Commonest head injuries are mild and superficial; and are caused by falls and road traffic accidents. Most head injury patients are children.

\section{Keywords}

Contusion, falls, head injury, traumatic brain injury 


\section{INTRODUCTION}

T raumatic head injuries constitute a major cause of morbidity and mortality worldwide. ${ }^{1-4}$ The burden of these injuries is particularly prominent in low- and middle-income countries (LMIC), which usually face more challenges regarding a higher prevalence of risk factors. ${ }^{3,5}$ The terminology regarding traumatic head injuries is a central issue in research. The term is commonly used synonymously with "traumatic brain injury" (TBI) by both researchers and physicians. ${ }^{6-8}$ A Working Group on Traumatic Brain Injury has defined TBI as "an alteration in brain function, or other evidence of brain pathology, caused by an external force"."

The clinical significance of TBls is the presence and development of more or less severe shortterm complications and sequelae. Among the most common severe short-term complications are intracranial haemorrhage associated with significant mortality, cerebrospinal fluid leakage and brain swelling leading to increased intracranial pressure.9,10 Related symptoms are headaches, seizures, and cognitive impairment. Long-term effects include post-concussion syndrome, as well as neurological and psychiatric sequelae. ${ }^{11-13}$ Most TBI patients recover within the first three months, but up to a third of the patients report symptoms persisting beyond six months. ${ }^{14,15}$

The diagnostics surrounding especially mild TBI have previously offered some difficulties. However, improved technological advances in magnetic resonance imaging and the development of biomarkers offer potentials for improving diagnostic accuracy in these situations. In the absence of sophisticated imaging tools, the term "head injury" is used in many hospitals, including the Emergency Department (ED) at Tribhuvan University Teaching Hospital (TUTH), Kathmandu, Nepal where this observational study was conducted. Thus, the study defined head injury as any trauma to the head including superficial injuries to the scalp or face.

The aim of the study was to characterise patients seeking medical care for traumatic head injuries at the ED of TUTH - focusing specifically on demographics, cause of trauma and type of injury as well as severity and outcome (admitted, discharged, death). Auxiliary aims were to examine possible correlations between age, trauma mechanisms and injury severity.

\section{METHODS}

In this descriptive study medical records dated from the $26^{\text {th }}$ of June to the $3^{\text {rd }}$ of October 2019 were searched manually. Patients diagnosed with intracranial hematomas, cerebral contusions, concussions/mild head injuries, fractures, soft tissue injuries (i.e. skin abrasions and contusions) and lacerations to the head and/or face were included. Patients with solely dental, ear, eye or cervical spine injuries were excluded.

Variables were collected using a predetermined pro forma. Patient demographics were registered, involving district of residence, divided into inside or outside Kathmandu Valley. Trauma mechanisms were allocated to road traffic accidents including pedestrian accidents, falls, struck by/against, violence/physical assault and other causes. Type of head injury was characterised. Injury severity was based on GCS-score determined at the triage or on doctors first assessment. A GCS of 13-15 was defined as mild, 9-12 as moderate and 3-8 as severe.

Patient demographics, type of injury and outcome of ED attendance were presented through descriptive statistics. To evaluate whether the age distribution among head injured patients solely depended on the age distribution of the population in Nepal or not, the patient age distribution was normalised by the overall Nepali population age. Differences in median age of patients regarding trauma mechanisms and injury severity were calculated using Mann-Whitney $\mathrm{U}$ independent samples median test.

The study was performed according to the Helsinki declaration. Prior to data collection, an ethical approval was granted from the Institutional Review Committee (IRC) at Institute of Medicine (IOM).

\section{RESULTS}

Out of 11,043 medical records searched, a total of 577 patients suffering from traumatic head injuries were identified and included, constituting $5.2 \%$ of all medical records examined. The ED served approximately 112 patients per day (11 043 medical records over 99 days, 111.5), six of which (577/99, 5.8) were head injuries. The mean age was 25.7 years and the median age was 23 years. More than one fourth of the patients (27.5\%) were under eleven years of age (Figure 1). In thirteen of the cases, age was not stated in the medical records.

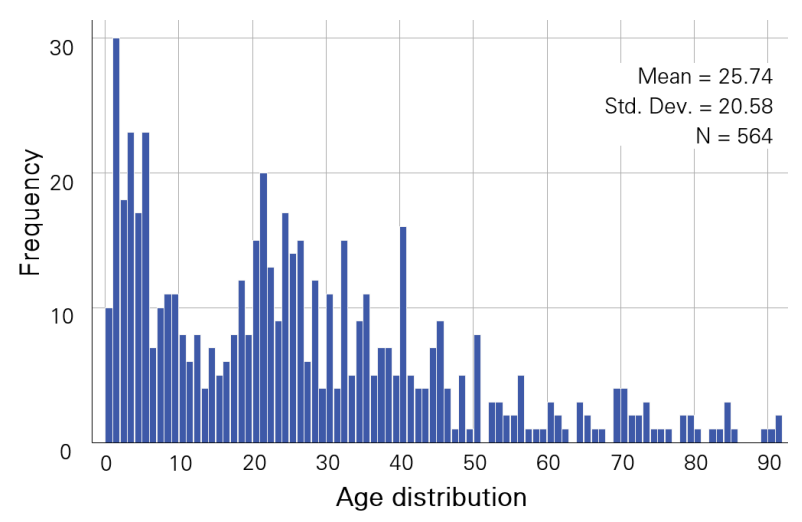

Fig 1. Age distribution histogram (Data missing for 10 patients) 
Table 1. Trauma mechanisms

\begin{tabular}{lc}
\hline \multicolumn{1}{c}{ Cause } & Number \\
\hline Falls & $282(48.9 \%)$ \\
Road traffic accident & $105(18.2 \%)$ \\
Violence/physical assault & $97(16.8 \%)$ \\
Struck by/against & $36(6.2 \%)$ \\
Other & $30(5.2 \%)$ \\
Unknown & $27(4.7 \%)$ \\
& Total \\
& $577(100.0 \%)$ \\
\hline
\end{tabular}

The patient age distribution was normalised by the overall population in Nepal, enabling the possibility of evaluating how the patient age distribution differed from the general population. The proportion of young patients in the study was lower compared to the general population in Nepal. Additionally, there was a peak among older patients compared to the general population.

The patients were predominantly male $168.8 \%$, $392 / 570)$. Gender was not stated in seven of the medical records. Patients from inside Kathmandu Valley constituted $55.1 \%$ (270/490) of the cases. A total of 87 patients had no or an unreadable district of residence logged in their medical record.

The most common cause of trauma was falls (48.9 $\%$, 282/577), followed by road traffic accidents $(18.2 \%, 105 / 577)$ and violence/physical assault (16.8 \%, 97/577). A small portion (6.2 \%, 36/577) of the patients had been struck by or against an object, e.g. while working at a construction site or while playing or practicing sports. The "other"category $(5.2 \%, 30 / 577)$ included animal bites and gunshots (Table 1). In 27 cases, no cause of trauma was noted in the medical records.

Falls had the broadest age distribution and the lowest median age (13 years), followed by struck

Table 2. Type of injuries

\begin{tabular}{lc}
\hline \multicolumn{1}{c}{ Diagnosis } & Number \\
\hline Concussion & $166(30.5 \%)$ \\
Superficial scalp injury & $160(29.4 \%)$ \\
Superficial face injury & $280(51.4 \%)$ \\
Superficial injury, unspecified & $13(2.4 \%)$ \\
Facial fracture & $12(2.2 \%)$ \\
Skull fracture & $17(3.1 \%)$ \\
Intracranial hematoma & $49(9.0 \%)$ \\
Cerebral contusion & $16(2.9 \%)$ \\
Pneumocephalus & $3(0.6 \%)$ \\
Other & $6(1.1 \%)$ \\
Unknown & $32(4.2 \%)$ \\
& $754^{*}(100.0 \%)$ \\
\hline
\end{tabular}

* Since a patient can receive several diagnoses, the total number of diagnoses exceeds the total number of patients.
Table 3. Injury severity according to GCS

\begin{tabular}{|c|c|c|}
\hline Severity & & Number \\
\hline Severe: GCS 3-8 & & $7(1.2 \%)$ \\
\hline Moderate: GCS 9-12 & & $17(2.9 \%)$ \\
\hline Mild: GCS 12-15 & & $408(70.7 \%)$ \\
\hline \multirow[t]{2}{*}{ Unknown } & & $145(25.1 \%)$ \\
\hline & Total & $577(100.0 \%)$ \\
\hline
\end{tabular}

by/against (14 years), other (20.5 years), road traffic accidents (24 years) and violence/physical assault (28.5 years). There was a significant difference in median age in patients suffering from falls compared to road traffic accidents $(p<0.001)$, violence/physical assault $(p<0.001)$ and other $(p=0.05)$. The median age of struck by/against differed significantly compared to violence/physical assault $(p=0.002)$, as well as road traffic accidents compared to violence/ physical assault $(p=0.01)$.

Superficial injuries were the most common $162.7 \%$, $453 / 722)$. Many of these injuries were facial (38.8\%, $280 / 722)$. Almost a quarter $(23 \%, 166 / 722)$ of the patients suffered from concussions, $6.8 \%$ (49/722) had intracranial hematomas and $4.1 \%(29 / 722)$ had skull or facial fractures. In 32 cases (4.2 \%, 32/754), no specific diagnosis was given further than e.g. "head trauma". These patients were categorised as "unknown" (Table 2). The "other"category includes penetrating brain injuries and diffuse axonal injuries.

Most of the injuries - 94.4\% (408/432) were classified as mild according to the GCS score (Table 3). Moderate injuries constituted $3.9 \%$ (17/432) of the cases and severe injuries $1.7 \%$ (7/432). In 145 of 577 cases, GCS score was not stated in the medical records. As shown in figure 2, mild head injuries had the lowest median age of 24 years. The

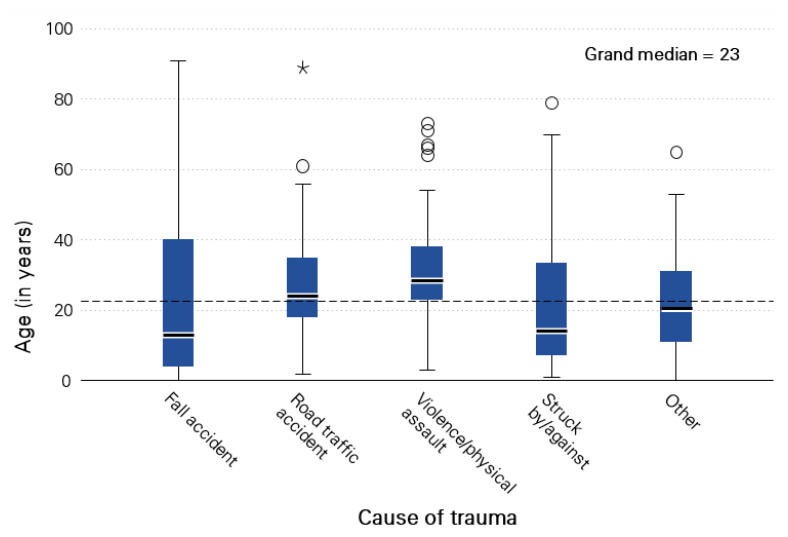

Fig 2. Median age by cause of trauma. The grand median is represented by the dashed line (23 years). Blue bars contain 50 per cent of the patients in each category. Lines below and above the blue bars each represent 25 per cent of the patients. The continuous line within each bar represent the median age in each category. Circles represent outliers. Stars represent extreme, far out values. The median age of falls was 13 years, struck by/against 14 years, other 20.5 years, road traffic accidents 24 years and violence/physical assault 28.5 years. 
median age of moderate injuries was 37 years and of severe injuries 36 years. Pairwise comparisons were not performed since the age distribution was the same across categories of severity and sample sizes of moderate and severe injuries were small. Nearly half of the patients $(45.2 \%, 238 / 527)$ were discharged from the ED or the observation ward after completed management (Table 4). Almost as many were discharged on patient request, absconded, or left against medical advice (41.9 per cent, 221/527), while $12.1 \%$ (64/527) were admitted for further management. Four patients died at the ED $(0.8 \%)$.

\section{DISCUSSION}

The purpose of this study was to establish age and gender distribution, trauma mechanisms and injury severity in head-injured patients attending the ED at TUTH, Kathmandu, Nepal. Traumatic head injuries accounted for $5.2 \%$ of all medical records searched. The ED served approximately 112 patients per day, around six of which were head injuries. Patients were predominantly male and around a fourth of the patients were under eleven years of age. Roughly half of the patients were from outside Kathmandu Valley. Most injuries were superficial and classified as mild according to the GCS-score. There was no significant difference in age across the categories of severity. The most common cause of trauma was fall accidents followed by road traffic accidents and the median age differed significantly depending on the cause of trauma. Only around a tenth (12.1 $\%$ ) of the patients were admitted to the hospital, $45.2 \%$ were discharged and $41.9 \%$ absconded, were discharged on patient request, or left against medical advice. Only four of the patients died at the ED $(0.8 \%)$.

The incidence of traumatic head injuries was roughly the same as in 2003, when a similar study was conducted by S. McClennan and C. Snider at TUTH. The age distribution was similar to the results previously found in studies from Nepal. ${ }^{16-19}$ In studies conducted in more developed countries, an incidence peak is usually seen in the elderly because of an increased risk of fall injuries. A corresponding peak was seen when normalising the age distribution by the overall population in Nepal, indicating that head injuries are common among the elderly when considering the small proportion of the population they comprise. However, because of different inclusion criteria and various categorisation of age groups, comparisons are somewhat unreliable. Additionally, the sample size among the elderly was small, meaning the proportion of injuries among elderly could be an overestimation.

In accordance with prior studies worldwide, males were exposed to head injuries at a higher rate than females. ${ }^{1,2}$ Anecdotally, due to gender roles
Table 4. Outcome of ED attendance

\begin{tabular}{lc}
\hline \multicolumn{1}{c}{ Outcome } & Number \\
\hline Discharged & $238(41.2 \%)$ \\
Admitted & $64(11.1 \%)$ \\
DOPR/LAMA & $170(29.5 \%)$ \\
Absconded & $51(8.8 \%)$ \\
Death & $4(0.7 \%)$ \\
Unknown & $50(8.7 \%)$ \\
& Total \\
\hline
\end{tabular}

*DOPR: discharged on patient request, LAMA: left against medical advice.

in Nepal (and globally), men are overrepresented in both high-risk occupations such as construction work and in perilous activities such as driving cars and riding motorcycles or bikes.

Half of the injuries were caused by falls. Similar studies on head trauma in Nepal and globally have repeatedly reported falls or road traffic accidents as the main cause of injury. ${ }^{2,5}$ Falls dominate among children and the elderly, while road traffic accidents and violence are common among young and middle aged adults. ${ }^{1}$ Though only a few studies have been published on head injuries in Nepal specifically, the results regarding cause of injury seem to coincide with prior studies. However, the proportion of road traffic accidents were considerably lower than previous studies with reported percentages of around $38-44 \% .{ }^{17-19}$ Possible explanations might be heavy rains causing severe flooding in Kathmandu during the study period or because of different inclusion criteria among these studies. The amount of injuries caused by violence or physical assaults agreed with results of prior studies from Nepal. ${ }^{18,19}$

Most patients had mild injuries according to the GCS-score (94.4\%). In previous studies from Nepal, mild injuries have composed around $80 \%$. 17,19 This discrepancy is most likely due to different inclusion criteria and the large amount of missing GCS-score data in this study. Roughly half of the patients were from outside Kathmandu Valley, likely due to deficient health care facilities in rural areas. Many patients are forced to travel long distances from the countryside on faulty roads to reach modern health care facilities.

Superficial injuries were the most common. A previous study from Nepal reported scalp lesions as the most common diagnosis, followed by fractures and cerebral contusions. ${ }^{18}$ In studies focusing on TBIs specifically, concussions and contusions are the most common diagnosis, followed by intracranial hematomas and fractures. ${ }^{11,16}$ The low contusion rate in this study might be explained by missing or noncomprehensive descriptions of CT-scan findings in the medical records. Additionally, not all patients underwent a CT-scan. The number of concussions 
and superficial injuries is an underestimation since many patients never seek medical help, perhaps due to financial and/or transportation issues. Therefore, the true impact of head injuries is likely much higher than estimated.

The median age differed significantly between the cause of trauma categories. The median age of falls (13 years) probably would have been higher if the expected lifespan in Nepal had been longer, due to an increased risk of falls among elderly. As expected, there was significant differences in median age in falls compared to several other categories due to the high number of young children suffering from falls. The large number of head-injured children presumably contributed to the lower median age of mild head injuries compared to moderate and severe injuries. A reasonable assumption would be that children are less exposed to high-energy trauma such as traffic accidents, and more commonly affected by mild injuries from e.g. falls.

Most patients either left against medical advice or were discharged either on patient request or directly from the observation ward. Possible reasons might be overcrowding at the ED, long waiting times, financial problems and congested wards. Some were advised to seek medical help at other hospitals due to the intensive care unit being full. In proportion to the rapidly growing population in Kathmandu, hospitals are increasingly challenged by overcrowding and must quickly adapt despite limited resources.

\section{CONCLUSION}

Mild and superficial head injuries are the commonest and are caused by falls and road traffic accidents. Most patients of head injuries are children.

\section{CONFLICT OF INTEREST}

None declared.

\section{ACKNOWLEDGEMENTS}

This article and the research behind it would not have been possible without the support from Sahlgrenska Academy at Gothenburg University and the Institute of Medicine, Tribhuvan University Teaching Hospital. Special thanks are due to the Sten A Olsson Foundation for the generous travel grant. We would also like to express deep gratitude to the helpful staff at the emergency department for their assistance with the data collection.

\section{REFERENCES}

1. Hyder AA, Wunderlich $C A$, Puvanachandra $P$, et al. The impact of traumatic brain injuries: A global perspective. NeuroRehabilitation. 2007;22(5):341-53.

2. James SL, Theadom A, Ellenbogen RG, et al. Global, regional, and national burden of traumatic brain injury and spinal cord injury, 1990-2016: a systematic analysis for the Global Burden of Disease Study 2016. The Lancet Neurology. 2019;18(1):56-87.

3. Dewan MC, Rattani A, Gupta $S$, et al. Estimating the Global Incidence of Traumatic Brain Injury. Journal of Neurosurgery. 2018;130(4):1080

4. Werner $C$, Engelhard K. Pathophysiology of traumatic brain injury. British Journal of Anaesthesia. 2007:99(1):4-9.

5. Reilly P. The Impact of Neurotrauma on Society: An International Perspective. Progress in Brain Research. 2007;161:3-9.

6. De Kruijk JR, Leffers $P$, Menheere PPCA, et al. Prediction of posttraumatic complaints after mild traumatic brain injury: early symptoms and biochemical markers. J Neurol Neurosurg Psychiatry. 2002;73(6):727-32.

7. Freire $F R$, Coelho $F$, Rhein $L$, et al. Cognitive rehabilitation following traumatic brain injury. Dementia \& neuropsychologia. 2011;5(1):17-25.

8. Menon DK, Schwab K, Wright DW, et al. Position Statement: Definition of Traumatic Brain Injury. Archives of Physical Medicine and Rehabilitation. 2010;91(11):1637-40.

9. Perel P, Roberts I, Bouamra O, et al. Intracranial bleeding in patients with traumatic brain injury: A prognostic study. BMC Emergency Medicine. 2009;9(1):15.

10. Ji-Woong O, So-Hyun K, Whang K. Traumatic Cerebrospinal Fluid Leak: Diagnosis and Management. Korean journal of neurotrauma. 2017;13(2):63-7.

11. Ahmed S, Venigalla H, Mekala HM, et al. Traumatic Brain Injury and Neuropsychiatric Complications. Indian journal of psychological medicine. 2017;39(2):114-21.

12. McAllister TW. Neurobehavioral sequelae of traumatic brain injury: evaluation and management. World psychiatry : official journal of the World Psychiatric Association (WPA). 2008;7(1):3-10.

13. Bazarian J J, Cernak I, Noble-Haeusslein L, et al. Long-term neurologic outcomes after traumatic brain injury. J Head Trauma Rehabil. 2009;24(6):439-51.

14. Sharp DJ, Jenkins PO. Concussion is confusing us all. Practical Neurology. 2015;15(3):172.

15. Evans RW. The postconcussion syndrome and the sequelae of mild head injury. Neurol Clin. 1992;10(4):815-47.

16. Bajracharya A, Agrawal A, Yam BR, et al. Spectrum of surgical trauma and associated head injuries at a university hospital in eastern Nepal. Journal of neurosciences in rural practice. 2010;1(1):2-8

17. Ghimire P, Yogi N, Acharya G. Management of Head Injury by General Surgeons in a General Hospital. Nepal Journal of Medical Sciences. 2012;1(1):19-22.

18. Adhikari K, Gupta MK, Pant AR, et al. Clinical Patterns and Computed Tomography Findings in Patients with Cranio-Cerebral Trauma in Tertiary Hospital in Eastern Nepal. Journal of Nepal Health Research Council. 2019;17(42):56-60.

19. McClennan S, Carolyn S. Head Injuries in Kathmandu, Nepal. Mc Master University Medical Journal. 2003;1(1):4-10. 